\title{
Granule formation in early infection
}

Although a detailed understanding of viral replication within, and transmission between, plant cells has begun to emerge in recent years, little is known about the early stages of viral infection. The bestcharacterized plant RNA virus is

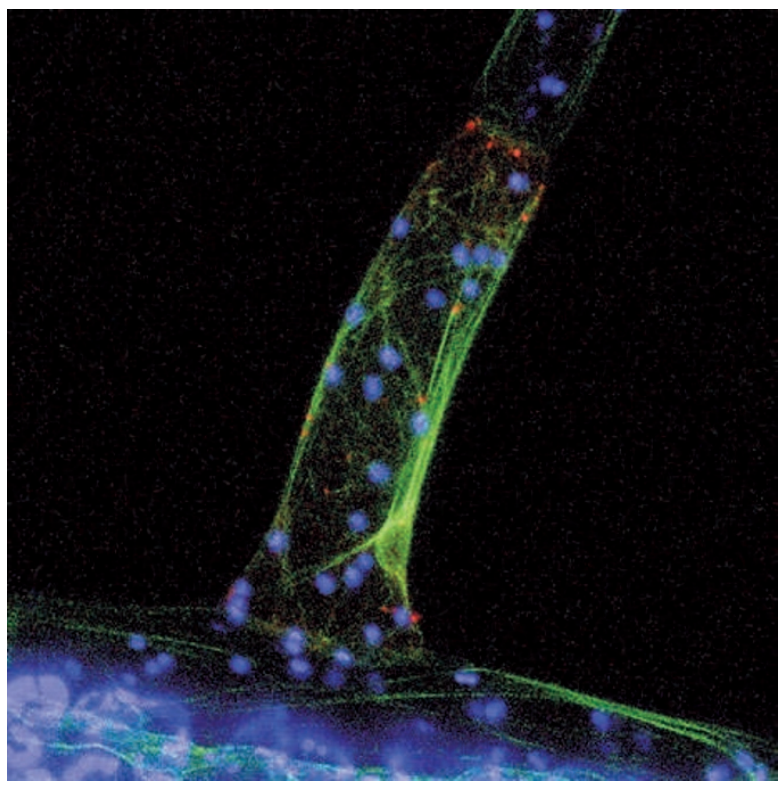

A trichome infected with tobacco mosaic virus. Cy3-vRNA granules are shown in red, GFP-labelled actin is shown in green and chloroplasts are shown in blue. Image courtesy of K. Oparka, University of Edinburgh, United Kingdom. tobacco mosaic virus (TMV), which is thought to infect plants through wounds at the cell surface. Now, Christensen and colleagues show that during infection the TMV genome forms granules that attach to the cortical endoplasmic reticulum (ER)-actin network prior to viral replication.

To track the viral genome in host cells, the authors used a fluorescent uridine $5^{\prime}$-triphosphate derivative to label the TMV viral RNA genome (Cy3-vRNA). They saw that during entry into a plant trichome, an outgrowing cell on the plant surface, Cy3-vRNA initially formed a diffuse cloud, which then condensed to form discrete granules in the cytoplasm. Granules were observed during infection with either intact or uncoated Cy3vRNA, indicating that the viral capsid protein was not required. The granules fused over time to form larger, motile granules. Comparison with GFP-tagged markers for particular cellular structures showed that movement of the Cy3-vRNA granules occurred along the cortical ER network. Furthermore, the actin inhibitors cytochalasin and latrunculin arrested granule movement on the ER-actin network, which showed that movement required actin filaments.

Viral RNAs have a 5'-methylguanosine cap, which is important for efficient translation and is thought to protect the viral RNA from digestion by exonucleases. Christensen and colleagues found that Cy3-vRNA that lacked the cap structure failed to form granules, and instead remained as a diffuse cloud.

This study is the first to document granule formation by a plant viral RNA and suggest that the viral RNA cap structure is essential for this process. Further work is necessary to determine the precise mechanism that underlies this pathway and identify any other host or viral factors involved.

Andrew Jermy

ORIGINAL RESEARCH PAPER Christensen, N. et al. The $5^{\prime}$ cap of tobacco mosaic virus (TMV) is required for virion attachment to the actin/ER network during early infection. Traffic 11 Feb 2009 (doi:10.1111/j.1600-0854.2008.00889.x) 